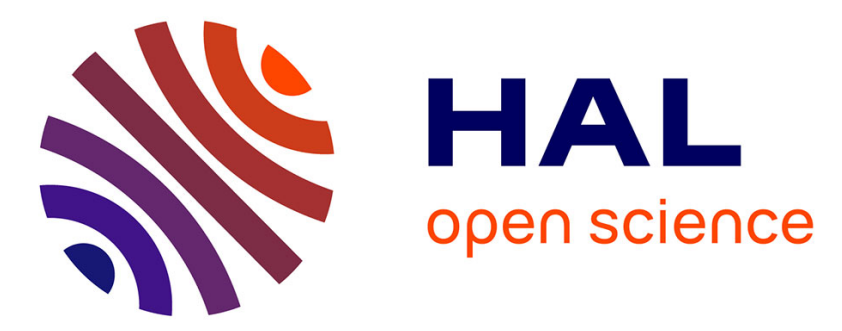

\title{
Investigating the effect of alumina shaping on the sorption properties of promising metal-organic frameworks
}

Paul Iacomi, U-Hwang Lee, Anil H Valekar, Jong-San Chang, Philip Llewellyn

\section{- To cite this version:}

Paul Iacomi, U-Hwang Lee, Anil H Valekar, Jong-San Chang, Philip Llewellyn. Investigating the effect of alumina shaping on the sorption properties of promising metal-organic frameworks. RSC Advances, 2019, 9 (13), pp.7128-7135. 10.1039/c9ra00534j . hal-02127029

\author{
HAL Id: hal-02127029 \\ https://hal.science/hal-02127029
}

Submitted on 13 May 2019

HAL is a multi-disciplinary open access archive for the deposit and dissemination of scientific research documents, whether they are published or not. The documents may come from teaching and research institutions in France or abroad, or from public or private research centers.
L'archive ouverte pluridisciplinaire HAL, est destinée au dépôt et à la diffusion de documents scientifiques de niveau recherche, publiés ou non, émanant des établissements d'enseignement et de recherche français ou étrangers, des laboratoires publics ou privés. 
Check for updates

Cite this: RSC Adv., 2019, 9, 7128

Received 21st January 2019

Accepted 12th February 2019

DOI: 10.1039/c9ra00534j

rsc.li/rsc-advances

\section{Investigating the effect of alumina shaping on the sorption properties of promising metal-organic frameworks $\uparrow$}

\author{
Paul lacomi, (iD a U-Hwang Lee, ${ }^{\text {bc }}$ Anil H. Valekar, ${ }^{\text {bc }}$ Jong-San Chang ${ }^{\text {bd }}$ \\ and Philip L. Llewellyn (iD*a
}

\begin{abstract}
Three promising MOF candidates, UiO-66(Zr), MIL-100(Fe) and MIL-127(Fe) are shaped through granulation with a $\rho$-alumina binder. Subsequently, changes in the surface characteristics and adsorption performance are evaluated through adsorption microcalorimetry at $303 \mathrm{~K}$ with several common probes $\left(\mathrm{N}_{2}, \mathrm{CO}_{2}, \mathrm{CO}\right.$, $\mathrm{CH}_{4}, \mathrm{C}_{2} \mathrm{H}_{6}, \mathrm{C}_{3} \mathrm{H}_{8}, \mathrm{C}_{3} \mathrm{H}_{6}$ and $\left.\mathrm{C}_{4} \mathrm{H}_{10}\right)$, generating a detailed picture of adsorbate-adsorbent interactions. Vapour adsorption experiments with water and methanol were further used to gauge changes in hydrophobicity caused by the addition of the alumina binder. Upon shaping, a decrease in gravimetric capacity and specific surface area is observed, accompanied by an increased capacity on a volumetric basis, attributed to densification induced by the shaping process, as well as a surprising lack of pore environment changes. However, the magnitude of these effects depends on the MOF, suggesting a high dependence on material structure. Out of the three materials, MIL-127(Fe) shows the least changes in adsorption performance and is highlighted as a promising candidate for further study.
\end{abstract}

\section{Introduction}

Research into the synthesis and application of metal-organic frameworks (MOFs) has been on the rise ever since their emergence in the nineties. The diverse range of structures and properties which can be attained through MOF chemistry has the potential to revolutionise areas such as separation, catalysis and gas storage. Moreover, due to their hybrid properties, MOFs can find novel uses in other fields such as electronics, biomedicine and micromechanics. ${ }^{1}$ Despite the great industrial interest in these metamaterials, practical applications have lagged behind academic research, seen as a result of the complexity of mass manufacturing and integration of MOFs into current processes and workflows.

In order to use an adsorbent in an industrial setting such as the beds and columns common in PSA (pressure swing adsorption) and TSA (temperature swing adsorption) processes, a pelletized form of the material is required. The shaping process is needed not just for containing the small particles, but

${ }^{a}$ Aix-Marseille Université, CNRS, MADIREL UMR 7246, 13397 Marseille, France. E-mail: philip.llewellyn@univ-amu.fr

${ }^{b}$ Research Group for Nanocatalysts, Korea Research Institute of Chemical Technology (KRICT), Daejeon 305-600, Korea

'Department of Green Chemistry, University of Science and Technology (UST), 217 Gajeong-Ro, Yuseong, Daejeon 305-350, Korea

${ }^{d}$ Department of Chemistry, Sungkyunkwan University, Suwon 440-476, Korea

$\dagger$ Electronic supplementary information (ESI) available: One pdf file with all referenced supporting information. See DOI: 10.1039/c9ra00534j also to impart the resulting pellet with both a high enough mechanical resistance to withstand the stresses involved in high flow, and a hierarchical structure which promotes rapid mass transfer while preventing large pressure drops. ${ }^{2}$ In an ideal setting, this process has limited effects on the properties of the material. The kinetics of adsorption are generally improved by the multi-scale pore size distribution afforded through shaping. Capacity per mass of pellet is expected to decrease due to the addition of a non-porous component, but the difference should simply be proportional to the amount of binder added and should not arise from effects such as pore blocking by the binder material. Furthermore, the densification effect is expected to lead to higher capacity on a volume basis of adsorbent. Finally, binder addition should not influence the chemical properties of the adsorbent, and thus preserve the prototypical interactions with the adsorbate. With a judicious choice of binding material, the resulting pellet should outperform the powder.

The shaping process is therefore a crucial step towards the large-scale use of an adsorbent material. Even for commonplace adsorbents such as carbons and zeolites, the optimum binding material and the shaping process itself are the subject of extensive research. Often, the procedure is tailored for individual materials and applications. For carbons, binders such as pitch, polymers (CMC, PVA) or even non-porous carbon black are commonly used. ${ }^{3}$ Often, a combination of binders is used, each with a different purpose during the pelletization process. ${ }^{4}$ The process itself consists of extrusion of the particle-binder slurry and then hardening either through temperature, cross- 
linking or chemical treatment. Other methods, such as spraydrying or granulation can similarly be used. ${ }^{5}$ For zeolites, inorganic binders are more prevalent, with silica, alumina and clay binders commonly used. It has been shown ${ }^{6,7}$ that the choice of binder can introduce large property variations, ranging from loss of porosity and structure to the enhancement of the desired reactivity and selectivity through changes in the acid site density or ion migration.

The shaping of MOFs has been attempted with a wide range of binders and methods. Methods such as granulation, spraydrying or extrusion have all been successfully employed to create MOF pellets. ${ }^{8}$ Monoliths have also been shown to be an effective way for shaping purposes, either through impregnation $^{9}$ or through support on alumina. ${ }^{10}$ Surprisingly, compression $^{\mathbf{1 1}}$ or even simple air drying of MOF slurries ${ }^{12}$ have also shown good results. The connection between MOF and binder is also of crucial importance for membranes. The MOF-polymer interface has been shown to be subject to a complex interplay of interactions between the organic chains and the crystal surfaces. ${ }^{13}$ These effects can be striking enough to warrant further research into MOF-polymer hybrids, ${ }^{\mathbf{1 4}}$ with the aim of combining the unique attributes of both materials.

In a previous study ${ }^{\mathbf{1 5}}$ the impact of PVA shaping on a series of MOFs was analysed. It was shown that the binder introduced some specific effects, such as a protection effect on the reduction of $\mathrm{Fe}^{3+}$ to $\mathrm{Fe}^{2+}$ in MIL-127(Fe), as well as a curious gating effect seen on butane adsorption on MIL-100(Fe), possibly due to polymer chains covering pore entrances. Otherwise, the shaping did not impact performance of the MOFs, with almost no capacity loss on a mass basis. Unfortunately, the use of a polymer limited the activation temperature of the samples to a maximum of $150{ }^{\circ} \mathrm{C}$.

In this work we have selected the same series of "topical" MOFs and have investigated the influence of a different binding agent ( $\rho$-alumina) during granulation. After verifying the thermal stability and specific surface area of the resulting pellets, several probes are used to observe potential differences between the two forms. Adsorption manometry combined with microcalorimetry is used to measure the isotherms of $\mathrm{N}_{2}, \mathrm{CO}_{2}$, $\mathrm{CO}, \mathrm{CH}_{4}, \mathrm{C}_{2} \mathrm{H}_{6}, \mathrm{C}_{3} \mathrm{H}_{8}, \mathrm{C}_{3} \mathrm{H}_{6}$ and $\mathrm{C}_{4} \mathrm{H}_{10}$, together with the corresponding differential enthalpy of adsorption at $303 \mathrm{~K}$. Separately, adsorption of water and methanol at $298 \mathrm{~K}$ is used to characterise potential surface changes in the studied MOFs afforded by the inclusion of the binder.

\section{Materials and methods}

\subsection{Materials}

The three MOFs selected for this study have been chosen due to their stability, as well as good adsorption performance. UiO$66(\mathrm{Zr})$ and its derivatives are well known due to their high thermal and chemical stability. ${ }^{\mathbf{1 6}}$ It has also shown promise in use for gas adsorption applications. ${ }^{17}$ It is composed of $\mathrm{Zr}_{6}$-Oxo clusters connected with benzene dicarboxylate (BDC) linkers to form a face-centered cubic framework. It contains a threedimensional arrangement of micropores with each centric octahedral cage surrounded by eight corner tetrahedral cages (with diameters of approximately $1.1 \mathrm{~nm}$ and $0.8 \mathrm{~nm}$ respectively) and connected through narrow triangular windows of approximately $0.6 \mathrm{~nm}$. MIL- $100(\mathrm{Fe})$ is a MOF which uses the benzene tricarboxylate (BTC) linker in conjunction with trimeric iron(III) octahedral clusters. ${ }^{18}$ The framework assembles in hybrid supertetrahedra which leads to very large pores: a $2.5 \mathrm{~nm}$ cage and a $2.9 \mathrm{~nm}$ cage. These pores are accessible through windows of $0.55 \mathrm{~nm}$ and $0.86 \mathrm{~nm}$ respectively. The iron trimers are coordinated with anions and have shown a propensity to partially reduce to a divalent $\mathrm{Fe}^{2+}$ state, exposing an open metal site in the process. ${ }^{19}$ The last material, MIL-127(Fe), originally reported by Liu et $a .^{20}$ is a MOF built from the same Fe(III) octahedra trimers as MIL-100(Fe), but using the $3,3^{\prime}, 5,5^{\prime}$-azobenzenetetracarboxylate (TazBz) linker, to produce a framework with the soc topology. This material has shown promise for large-scale synthesis. ${ }^{21}$ Furthermore, it presents two types of pores with different surface characteristics: a hydrophobic 1D channel system of $0.6 \mathrm{~nm}$ and more hydrophilic cages of $1 \mathrm{~nm}$ diameter, accessible through narrow $0.3 \mathrm{~nm}$ windows. This MOF has been shown to be of interest for multiple applications such as catalysis or $\mathrm{CO}_{2}$ capture. ${ }^{22}$ The structures of the three materials can be seen in Fig. 1. The synthesis pathway of these materials has been previously reported, ${ }^{23}$ and further details can be found in the ESI. $\dagger$

\subsection{Shaping procedure}

The shaping of the samples was performed using a wet granulation method detailed elsewhere. ${ }^{23}$ In brief, the MOF powder was mixed with the previously prepared mesoporous $\rho$-alumina, with water added as the dispersing medium. The resulting mixture was shaped into beads using a hand-made pan granulator. During the process, the spheres were sprayed with water until the desired size was obtained. The beads were then sieved and rolled using a roller machine to enhance their spherical shape. Finally, the prepared samples were dried at $303 \mathrm{~K}$ for $12 \mathrm{~h}$ to remove all residual solvent. The resulting beads were near spherical in shape, with a diameter between $2 \mathrm{~mm}$ to 2.5 $\mathrm{mm}$. The total amount of binder in the granules is $5 \%$ by weight. ${ }^{23}$

\subsection{Characterisation of powders and pellets}

The primary interest of the study is to observe the changes in adsorption properties between the powder and the shaped materials which would be relevant in an industrial setting. First,
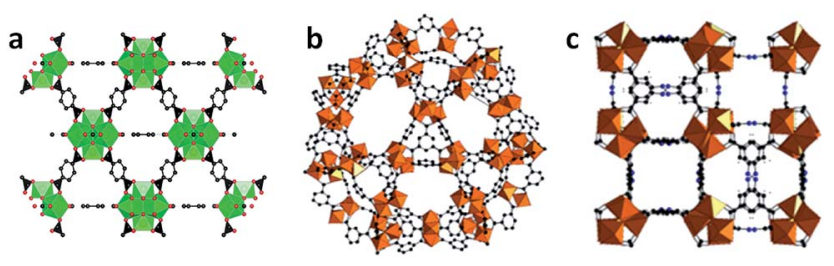

Fig. 1 The unit cell structures of the investigated MOFs: (a) UiO-66(Zr), (b) MIL-100(Fe) and (c) MIL-127(Fe). Zr polyhedra are displayed in green, Fe octahedra in brown, $\mathrm{C}$ in black, $\mathrm{O}$ in red, $\mathrm{N}$ in blue. Hydrogen atoms are omitted for clarity. 
the bulk density of the powder and pellets was measured to evaluate the level of densification afforded through the shaping process. Thermogravimetric analysis was then used to verify that the binder did not change the thermal stability of the materials. Specific surface area and pore volume were determined through nitrogen adsorption at $77 \mathrm{~K}$.

To observe any changes in adsorption energetics, isotherms and differential enthalpy curves were directly recorded through combined adsorption manometry and microcalorimetry. Even though the different contributions to the overall enthalpy curve cannot be decoupled from the individual sources, such as gasadsorbent interactions, gas-gas interactions or confinement effects, this technique is well suited for observing the effect of a process or treatment such as shaping on the properties of an adsorbent material. The isotherms of nine probe gases have been measured at $303 \mathrm{~K}: \mathrm{N}_{2}, \mathrm{CO}, \mathrm{CO}_{2}, \mathrm{CH}_{4}, \mathrm{C}_{2} \mathrm{H}_{6}, \mathrm{C}_{3} \mathrm{H}_{6}, \mathrm{C}_{3} \mathrm{H}_{8}$ and $n-\mathrm{C}_{4} \mathrm{H}_{10}$. The range of adsorbates chosen allows different effects to be investigated. The adsorption of saturated hydrocarbons with an increasing carbon number (C1-C4) is likely driven by non-specific interactions. Differences in the uptakes of these gases will point to loss of porosity or amorphisation. An asymmetric capacity loss with the larger molecules may suggest binder-induced size exclusion effects, such as particle coating, pore filling or pore obstruction. The other probes can be used to verify changes in specific interactions during adsorption. Carbon monoxide is a dipolar molecule which may highlight coordinatively unsaturated sites (CUS) generated through defects, reduction or open metal sites, due to its propensity for $\pi$ backbonding coordination. This type of electron transfer also can result in interactions with other systems with $\pi$ bonds such as alkenes and alkynes. Propylene is used as a probe gas for this purpose. Carbon dioxide is a quadrupolar molecule which will be strongly adsorbed in polar pore environments. Changes in the adsorption behaviour of $\mathrm{CO}_{2}$ will shed light on such surface changes and can even be used as a predictor of hydrophobicity. ${ }^{22}$ Finally, $\mathrm{N}_{2}$ is a staple adsorbent for material characterisation when used at $77 \mathrm{~K}$. The molecule is a slight quadrupole and has also been shown to chelate to some transitional metals in an analogue fashion to CO. It is important to note that, at $303 \mathrm{~K}$, adsorption of $\mathrm{N}_{2}, \mathrm{CO}$ and $\mathrm{CH}_{4}$ occurs in a supercritical regime.

To eliminate the influence of kinetic and diffusion effects on the results, care has been taken to allow time for complete equilibration of both pressure and calorimeter signal. After collecting the combined isotherm enthalpy data, three indicators have been chosen to best observe the effects of shaping: initial enthalpy of adsorption $\left(\Delta_{\text {ads }} h_{0}\right)$, initial Henry constant at zero loading $\left(K_{\mathrm{H}, 0}\right)$ and plateau molar adsorption capacity $\left(n_{\max }\right)$. The initial enthalpy of adsorption extrapolated at zero coverage is a measure of the interaction with highest energetic sites on the MOF surface. It has been calculated through a least square fitting of a logistic function on the differential enthalpy curve. Conversely, Henry's constant at zero loading is also an indication of adsorption before any lateral guest-guest interactions come into effect. $K_{\mathrm{H}, 0}$ was calculated by fitting a four coefficient virial adsorption model (eqn 1) to the isotherm.

$$
\ln \frac{n}{p}=\ln K_{\mathrm{H}, 0}+A n+B n^{2}+C n^{3}
$$

The last indicator, total molar capacity, was taken as the loading attained when the isotherm reached a plateau. In the case of probes where the plateau was outside the pressure range of the instrumentation ( $>50$ bar), the loading at the highest available pressure was considered as a suitable approximation. It is calculated on a mass basis and volume basis of adsorbent. The three key performance indicators (KPI) can then been compared for both the powder and shaped samples.

Finally, the influence of the binder on the hydrophobic character of the material may be of interest. Water is often found in small amounts in process conditions in the inlet stream, and has been shown to act as a competing adsorbate and be preferentially selected over the desired gas. ${ }^{24-26}$ Furthermore, as MOFs can sometimes be sensitive to hydrolysis, ${ }^{27,28}$ if the binder chosen can have an effect on the hydrophilicity of the adsorbent material it may allow for further control over this parameter. Due to its surface charges, alumina can be considered a hydrophilic substance, with a contact angle of $10^{\circ}$. It is expected that its addition may therefore increase the affinity of the resulting pellet towards hydrogen bonding. Here, water and methanol can serve as probes for small changes in surface properties. Therefore, adsorption isotherms with these vapours were recorded at $298 \mathrm{~K}$.

Details of the experimental procedure for each method can be found in the ESI. $\dagger$

\section{Results and discussion}

\subsection{Thermal stability and densification}

The process of shaping did not have any impact on the thermal stability of the investigated MOFs, as evidenced by the thermogravimetric curves (Fig. S1†). The primary mass loss occurs in a $10 \mathrm{~K}$ range for all powder-pellet pairs. The curves diverge after MOF degradation, owing to the addition of inert alumina. This is expected as the adsorbent makes up a lower percentage of total pellet mass. It is possible that degradation of surface alumina $\mathrm{OH}$ functionalizations may also contribute to a small mass loss throughout the entire temperature range in the granules. The measured bulk densities are shown in Table 1. A clear densification can be observed in all pellets, $48 \%$ for UiO66(Zr), 38\% for MIL-100(Fe) and 27\% for MIL-127(Fe).

Table 1 Properties of the studied powders and pellets

\begin{tabular}{lllll}
\hline MOF & Form & $\begin{array}{l}\text { Bulk density } \\
\left(\mathrm{kg} \mathrm{m}^{-3}\right)\end{array}$ & $\begin{array}{l}\text { BET surface } \\
\text { area }\left(\mathrm{m}^{2} \mathrm{~g}^{-1}\right)\end{array}$ & $\begin{array}{l}\text { Pore volume } \\
\left(\mathrm{cm}^{3} \mathrm{~g}^{-1}\right)\end{array}$ \\
\hline UiO-66(Zr) & Powder & 0.319 & 903 & 0.38 \\
& Pellet & 0.472 & 619 & 0.24 \\
MIL-100(Fe) & Powder & 0.216 & 1928 & 0.78 \\
& Pellet & 0.351 & 1451 & 0.59 \\
MIL-127(Fe) & Powder & 0.412 & 1413 & 0.76 \\
& Pellet & 0.526 & 1266 & 0.56
\end{tabular}




\subsection{Characterisation through nitrogen sorption}

Nitrogen sorption isotherms measured at $77 \mathrm{~K}$ have been measured on both powder and $\rho$-alumina pellets (Fig. S2 $\dagger$ ). The shapes of all isotherms are similar, with lower uptakes observed on the shaped materials. In both powders and pellets, the increased uptake after $0.9 p / p^{0}$ is a sign of condensation in very large pores or voids, which can be attributed to intra-pellet spaces and crystal agglomeration. In the MIL-127(Fe) pellets, a narrow hysteresis curve is seen, which closes at a $p / p^{0}$ of 0.5 . This curve corresponds to capillary condensation in a pore size of around $4 \mathrm{~nm}$, identical to the average pore size of the alumina binder. ${ }^{23}$ However, as this feature is present in both the pellet and powder isotherms, it cannot correspond to the binder and is therefore a likely consequence of particle agglomeration.

As predicted, the nitrogen accessible surface area of the shaped samples (Table 1) decreases compared to the corresponding powder. While in the case of MIL-127(Fe) the BET area is only $10 \%$ lower, for the MIL-100(Fe) and UiO-66(Zr) materials a larger drop is seen, of $25 \%$ and $31 \%$, respectively. A similar decrease can be seen in total pore volume (Table 1), with a $36 \%$, $23 \%$ and $26 \%$ loss seen in UiO-66(Zr), MIL-100(Fe) and MIL$127(\mathrm{Fe})$ respectively. The decrease in both surface area and micropore volume is unexpected for the amount of alumina binder added. The trends in surface area and pore volume also appear correlated with the increase in pellet density. It may therefore be possible that some structural degradation to have occurred during the pelletisation process, affecting MIL-127(Fe) the least and UiO-66(Zr) the most.

\subsection{Room temperature gas adsorption and microcalorimetry}

The complete calorimetry dataset measured, presented on a mass and volume basis, can be found in the ESI (Fig. S4-S27). $\dagger$ Results of note will be further discussed for each material.

3.3.1 UiO-66(Zr). The enthalpy curves on as-synthesised UiO-66(Zr) shows it to be a relatively homogenous material, with flat profiles being common. This is typical of this MOF, which has a pore environment without high energy adsorption sites. ${ }^{17}$ No large changes in either isotherm shape or enthalpy curve are visible whatever the probes used. Furthermore, the KPI graphs show very similar values for both initial Henry's constant (Fig. 2a) and initial enthalpy of adsorption (Fig. 2b). It is therefore apparent that this shaping process did not change the interaction of the adsorbate with the MOF surface. The maximum capacity graphs (Fig. 2c) show a more interesting trend. When using small adsorbates such as $\mathrm{N}_{2}, \mathrm{CO}_{2}$ and $\mathrm{CH}_{4}$, the shaped samples have a similar performance on a mass basis and, due to the densification process, better capacities on a volume basis. Starting with ethane, the maximum capacity difference starts to increase, with lower $n_{\max }$ as molecule size increases. On hydrocarbons with a carbon number of 3 and 4, both mass basis and volume basis capacity are decreased compared to the original powder. This size exclusion effect could be explained by the coating of crystal surfaces and pore entrances with the alumina binder. It could also be argued that instead of size exclusion, the effect is due to the pressure range at which isotherms are measured, and low molecular weight
UiO-66(Zr) initial Henry constant

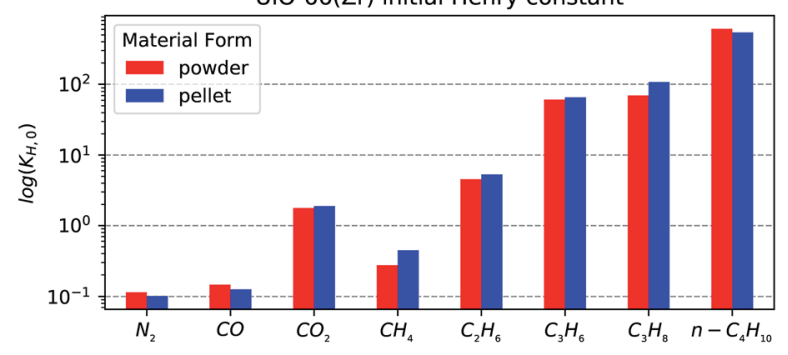

UiO-66(Zr) initial enthalpy of adsorption

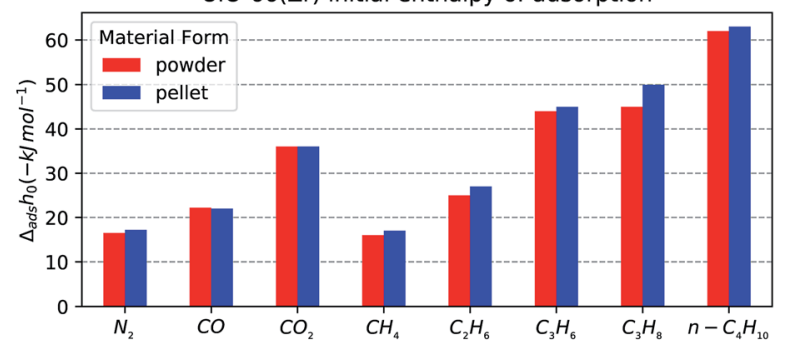

UiO-66(Zr) difference in capacity

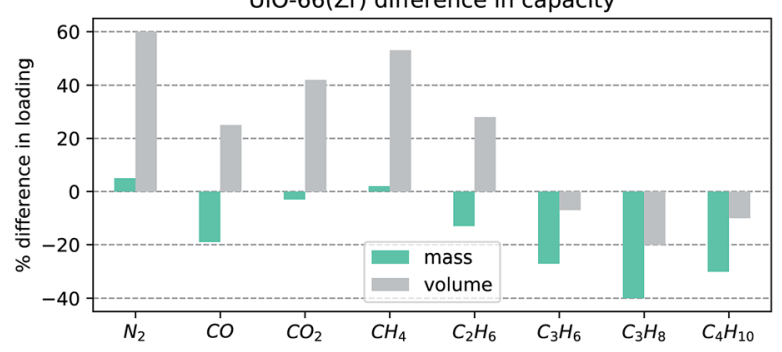

(c)

Fig. $2 \mathrm{KPI}$ extracted from the UiO-66(Zr) adsorption dataset with (a) logarithmic initial Henry constant (b) initial enthalpy of adsorption and (c) change in adsorption maximum capacity from the powder to the alumina shaped version on a mass and volume basis.

gases will diverge at higher pressures as the pores are filled. A counterargument for this hypothesis is that in the case of $\mathrm{CO}_{2}$, the plateau is reached with no differences between the powder and the pellet.

Carbon monoxide is an apparent outlier to this trend, with a decreased maximum capacity and a small molecular size. However, when observing the isotherms directly (Fig. S6†) experimental error can not be ignored, considering the low amount adsorbed and the good overlap in the enthalpy curves. Overall, the shaping performance of $\mathrm{UiO}-66(\mathrm{Zr})$ is reasonable, as long as only small adsorbates are used.

3.3.2 MIL-100(Fe). The enthalpy profiles on the MIL$100(\mathrm{Fe})$ powder are less homogenous than the those of UiO$66(\mathrm{Zr})$. Some effects can be seen with probes which may interact with partially reduced $\mathrm{Fe}(\mathrm{II})$ sites, such as carbon monoxide and propylene (Fig. 3). Indeed, when comparing the initial Henry constants and enthalpy of adsorption for CO and $\mathrm{C}_{3} \mathrm{H}_{6}$, they are higher than the values obtained on UiO-66(Zr). With an initial enthalpy of adsorption for $\mathrm{CO}$ of around $45 \mathrm{~kJ} \mathrm{~mol}^{-1}$, the value falls into the range of previous results ${ }^{19}$ for interactions with $\mathrm{Fe}(\mathrm{II})$ CUS.

Comparing the KPI for the powder and shaped MIL-100(Fe), there are no statistically significant differences between the two. 

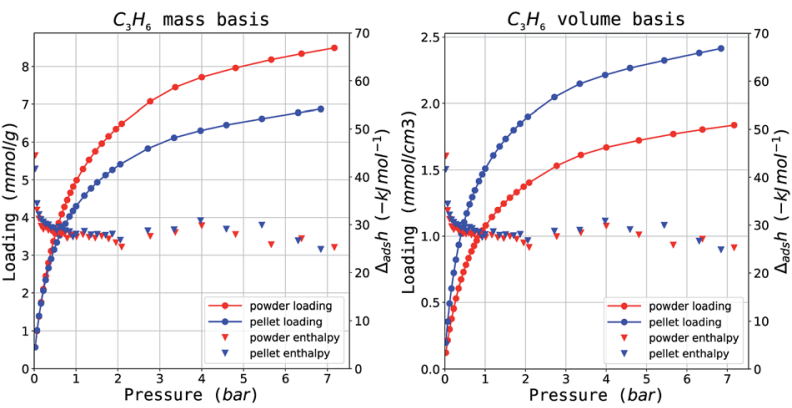

Fig. 3 Propylene isotherms on MIL-100(Fe) on a (left) mass and (right) volume adsorbent basis. Results are in red for the powder and blue for the shaped samples.

It is likely that on activation at a higher temperature $\left(250^{\circ} \mathrm{C}\right)$, the percentage of iron trimers which would undergo reduction will increase and a more pronounced CUS interaction could be observed. The maximum loading differences (Fig. 4c) of MIL$100(\mathrm{Fe})$ show a very similar behaviour. On all probes tested, a fixed capacity loss of between $10-20 \%$ can be seen on a mass basis. However, the increase in density afforded by compression

(a)

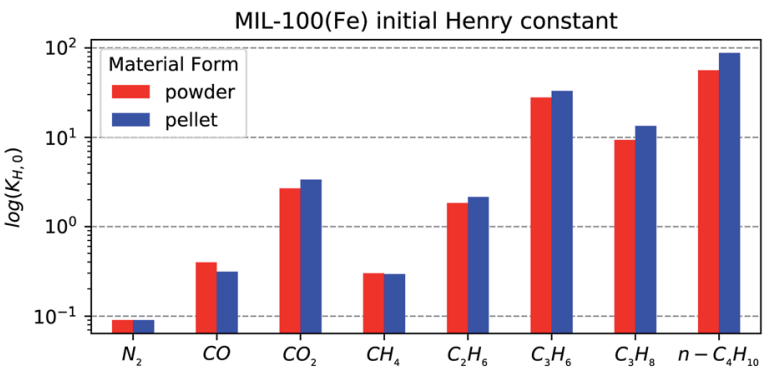

MIL-100(Fe) initial enthalpy of adsorption

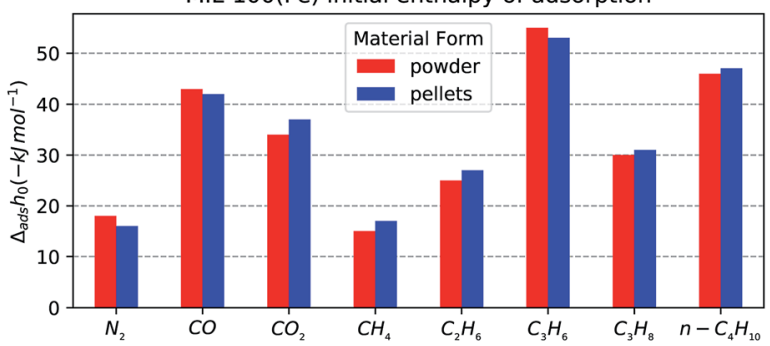

(b)

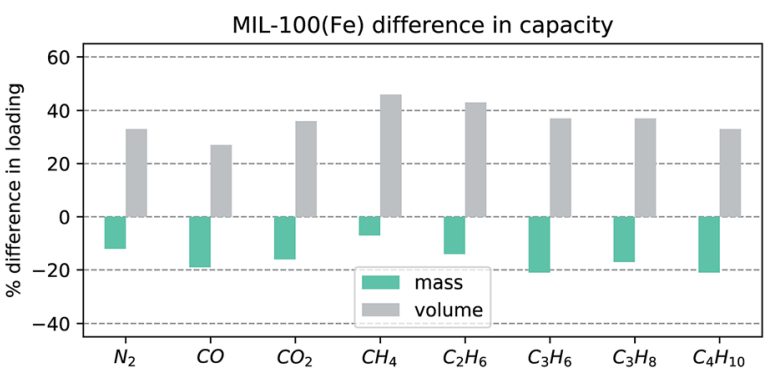

(c)

Fig. $4 \mathrm{KPI}$ extracted from the MIL-100(Fe) adsorption dataset with (a) logarithmic initial Henry constant (b) initial enthalpy of adsorption and (c) change in adsorption maximum capacity from the powder to the alumina shaped version on a mass and volume basis. during pelletisation leads to a compensation in performance as can be seen directly in the isotherms on mass and volume material basis in Fig. 3a and b respectively.

3.3.3 MIL-127(Fe). The isotherms on the as-synthesised MIL-127(Fe) could be expected to show similar initial slopes as MIL-100(Fe), due to the presence of the same iron trimesate moieties, although with a sharper uptake as a result of the smaller pores. Enthalpy profiles are also influenced by the CUS interactions, leading to higher initial enthalpies of adsorption on $\mathrm{CO}$ and $\mathrm{C}_{3} \mathrm{H}_{6}$. An overall increase in the enthalpy of adsorption at higher loadings is seen throughout the probe series, for example on butane (Fig. 5a). Due to the bimodal pore distribution of the MIL-127(Fe) structure, it is likely that adsorption first commences in the small $(\sim 0.8 \mathrm{~nm})$ channels and then, at higher pressures, intrusion into the larger cagetype pores is possible through the $\sim 0.3 \mathrm{~nm}$ narrow apertures. The confined cages have an increased interaction with the molecule which leads to the higher enthalpy values.

When comparing the powder and the pellet variants with respect to initial Henry's constant, a large difference in $K_{\mathrm{H}, 0}$ on $\mathrm{CO}$ is observed. The value of the initial enthalpy of adsorption does not follow the same pattern. However, visual inspection of the enthalpy curve in Fig. 5b shows that the energy of adsorption corresponding to interactions with the more active sites is maintained for a larger percentage of the total coverage. This points to the higher preponderance of such sites in the powder variant. A similar offset can be seen in the propylene enthalpy at very low pressures, through it is not reflected in the shape of the isotherm. The weaker complexation strength and the larger size of the molecule likely limits the effect. As for the underlying explanation behind the isotherm divergence, it may be that the alumina binder acts as protection against the generation of iron(II) during thermal activation with this particular structure. No other differences are seen between the two forms on either Henry constant and initial enthalpy of adsorption.

The capacity comparison in Fig. 6c paints an interesting picture. For most probes there is no change in maximum loading, suggesting no structural degradation or pore filling. Two outliers are apparent: carbon monoxide and butane. The decrease in capacity on $\mathrm{CO}$ can be explained through the aforementioned changes in active site prevalence. The drop in butane cannot be a consequence of the same effect as there is

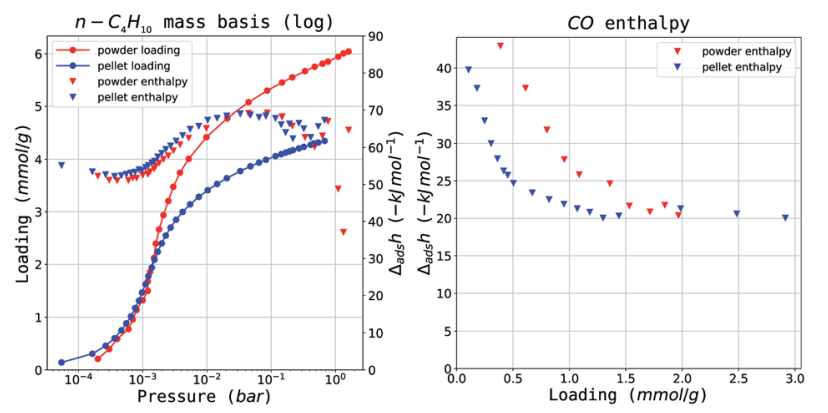

Fig. 5 Selected isotherms from the MIL-127(Fe) dataset: (left) $\mathrm{C}_{4} \mathrm{H}_{10}$ adsorption isotherms and (right) $\mathrm{CO}$ enthalpy curves. Results are in red for the powder and blue for the shaped samples. 
(a)

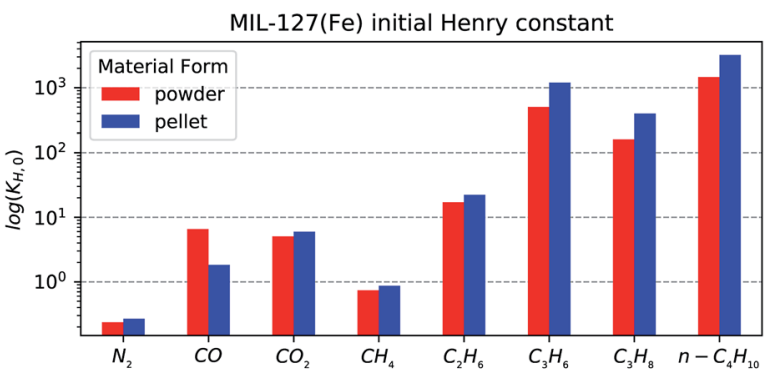

(b)

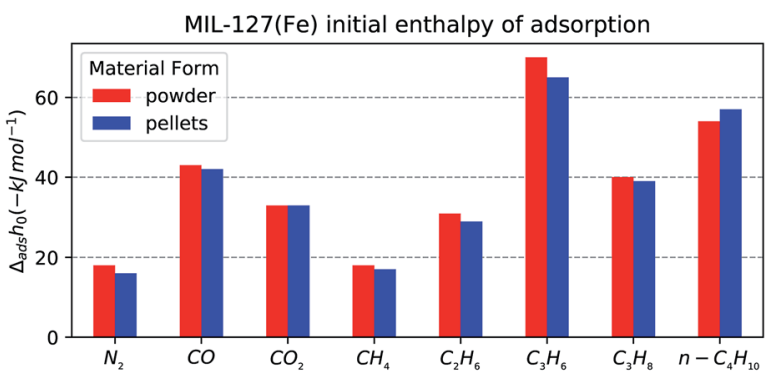

(c)

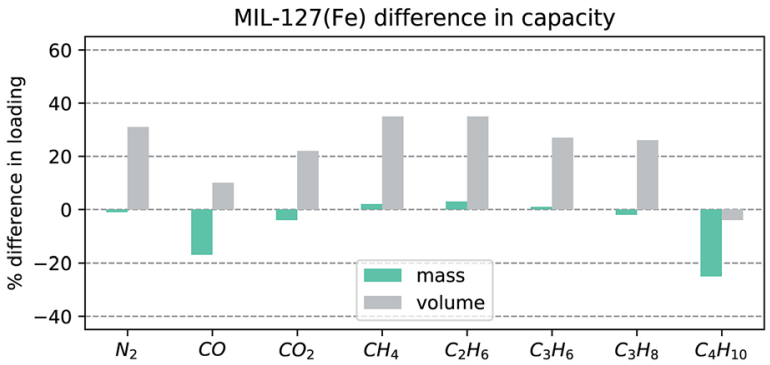

Fig. $6 \mathrm{KPI}$ extracted from the MIL-127(Fe) adsorption dataset with (a) logarithmic initial Henry constant (b) initial enthalpy of adsorption and (c) change in adsorption maximum capacity from the powder to the alumina shaped version on a mass and volume basis.

a perfect overlap in the enthalpy curves as seen in Fig. 5a. Therefore it likely better explained through a size exclusion effect similar to UiO-66(Zr). Overall, MIL-127(Fe) shows excellent performance after alumina shaping, with almost no capacity loss, as long as carbon monoxide or butane adsorption are not the required probes, where specific effects come into play.

\subsection{Vapour adsorption}

The effects of shaping with $\rho$-alumina as a binder are so far more subtle than the changes encountered when using other binders. ${ }^{15}$ As such the characterisation was extended using adsorption of vapours at room temperature.

Several isotherm features may highlight changes in material hydrophilicity: the slope of the isotherm in the low relative pressure region $\left(p / p^{0}<0.3\right)$ and filling steps in the isotherm. Isotherm slope at low pressures is representative of the initial interactions with the surface, as discussed in the previous section. The pressure at which condensation occurs in the pores of the material (underlined by a sharp increase in the isotherm) depends on the size of the pore but also on pore environment and guest-guest interactions. Finally, hysteresis in the adsorption isotherm may also be an indication of the nature of pores. The measured isotherms on water and methanol can be found in Fig. 7. Initial Henry constants $K_{\mathrm{H}, 0}$ have been calculated and are displayed in Fig. 8.

3.4.1 UiO-66(Zr). On the parent UiO-66(Zr), the water isotherm shows a slow initial uptake, indicating a hydrophobic surface, followed by a small step at $0.3 p / p^{0}$. While little is adsorbed on the MOF before this step, its presence at a low relative humidity is indicative of intrinsic defects in the framework. ${ }^{29}$ Complete saturation takes place around $0.9 p / p^{0}$. A wide hysteresis curve can be seen, which does not fully close, even at low pressures. Both the saturation step and the condensation may be attributed to agglomeration of crystals and interparticle voids. The methanol isotherm has the same features as the water one, with the initial step shifted at a much lower partial pressure $\left(0.1 p / p^{0}\right)$. It is likely that the methyl group interacts with the hydrophobic surface, thus permitting pore filling at lower pressures, also evidenced through the higher
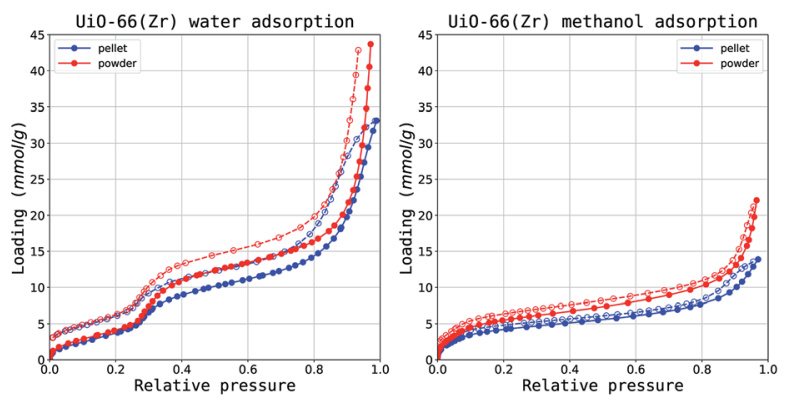

(a)
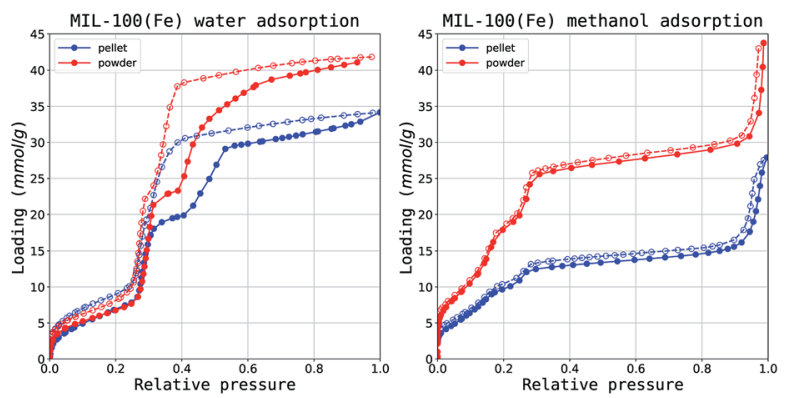

(b)
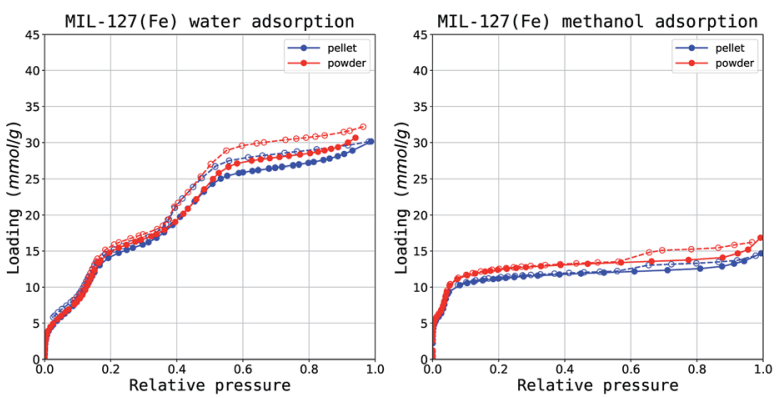

(c)

Fig. 7 Water (left) and methanol (right) adsorption isotherms (a) UiO66(Zr), (b) MIL-100(Fe) and (c) MIL-127(Fe). The powder and $\rho$-alumina isotherms are displayed in red and dark blue respectively. 


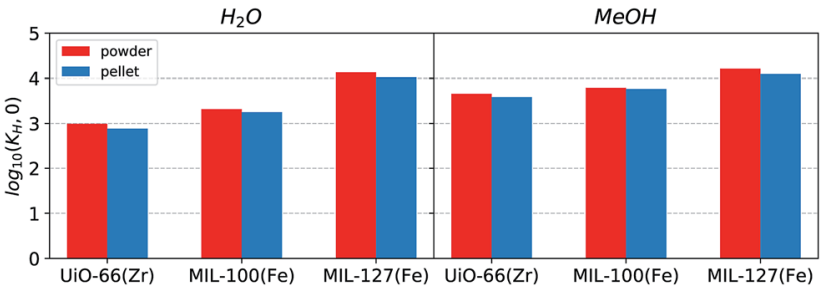

Fig. 8 Calculated initial Henry constant for the adsorption isotherms of (left) water and (right) methanol. The powder and $\rho$-alumina values are displayed in red and dark blue respectively.

Henry constant when as to water. When comparing the powder and the pellet variants, the general shape of the isotherm remains the same with both water and methanol. Initial interactions with the surface are also identical, as evidenced by the overlap in the low pressure region and through the calculated Henry constants. The isotherms begin to diverge after the condensation step, where the plateau attained is lower for the pellet than the powder for both vapours.

3.4.2 MIL-100(Fe). The water isotherm on the powder MIL$100(\mathrm{Fe})$ material shows a higher initial uptake, and two steps at 0.3 and $0.5 p / p^{0}$. At low pressures, adsorption takes place on metal sites in the large cages, with clusters formed around these hydrophilic sites. The two steps likely correspond to the successive filling of the $2.5 \mathrm{~nm}$ and the $2.9 \mathrm{~nm}$ pores. The adsorption and desorption branches show a hysteresis loop formation, associated with the condensation in the largest mesoporous spherical pore. The methanol isotherm on the same parent MOF still shows two condensation steps, which have been shifted at a lower pressure. There is no longer any hysteresis present, an indication that the critical pore radius for its development is not attained when using methanol. When comparing the powder isotherms with their shaped counterparts, while the general features of the isotherm remain the same, large shifts are seen in the adsorbed amounts. Adsorption of $\mathrm{H}_{2} \mathrm{O}$ proceeds in an identical fashion until the condensation step at $0.3 p / p^{0}$, where the alumina binder variant diverges to reach a plateau about $5 \mathrm{mmol} \mathrm{g}^{-1}$ smaller than the powder isotherm. The shape of the hysteresis loop also changes slightly, with a sharper slope on the adsorption branch that closes before $0.5 p / p^{0}$. Some changes can also be seen on the methanol adsorption isotherm, where the condensation steps are shifted towards lower pressures. These capacity drops are consistent with the values obtained with nitrogen at $77 \mathrm{~K}$ and room temperature calorimetry experiments. However, no influence is seen on the initial filling behaviour as the Henry's constant in this region confirms that low pressure interactions do not change.

3.4.3 MIL-127(Fe). On this MOF powder, the water isotherm suggests the most hydrophilic surface, with the highest initial slope out of the three materials studied. The isotherm has two condensation steps, one at low relative humidity $0.15 p / p^{0}$, which corresponds to the filling of the hydrophilic pore, and a condensation step situated at $0.5 p / p^{0}$ inside the more hydrophobic micropore. No hysteresis is observed. As a striking difference from water behaviour, methanol adsorption leads to completely filled pores at below 0.15 relative pressure. By examining the logarithmic form of the isotherms (Fig. S29†) a steep slope at low pressures is evident. It is likely that the larger size of the methanol molecule is much more affected by confinement in the hydrophilic pore. The same effect, combined with the increased affinity for the methyl group of this probe is likely responsible for shifting the secondary condensation step at lower pressures. The similarities between both variants of material confirm the previously observed results. The isotherms overlap almost fully, with a slight decrease in maximum uptake when shaped. The binder has not affected the interaction with the guest, as evidenced by the near-identical initial Henry constant.

\subsection{Long term stability of shaped samples}

The alumina shaped pellets should retain their properties and porosity even after exposure to humidity for an extended time. To this end, the samples used in the vapour adsorption experiments from the previous section were left exposed to air at ambient conditions for 12 months on a laboratory countertop. A new set of nitrogen sorption isotherms were recorded after this time (Fig. S3†). There is an almost perfect overlap between the two isotherms, confirming the long-term stability of the pellets.

\section{Conclusions}

The process of granulation with $\rho$-alumina does not, in general, have a strong impact on the surface chemistry of the three MOF materials chosen here. It can, however, lead to possible loss of usable adsorption capacity in the resulting pellets, corroborated by the decrease in BET area and pore volume. Interestingly, each studied material reacts differently to this shaping method: UiO-66(Zr) has a higher loss in capacity with larger molecular probes, MIL-100(Fe) has a decrease of 10-20\% across all gases and MIL-127(Fe) matches loading on a mass basis except on $\mathrm{CO}$ and $\mathrm{C}_{4} \mathrm{H}_{10}$. The shaping also induces a densification which, in almost all cases, leads to a better performance on a volumetric basis. However, the influence of the alumina binder on mass and heat transport is not known and should be investigated further. In particular, the iron-based MIL-127(Fe) is highlighted as a promising candidate for further use in gas storage or separation applications, as it exhibits nearly identical adsorption behaviour after granulation. Overall, the process of alumina shaping is a promising method of preparing MOFs for gas-related applications in separation and storage, but care should be taken to not generalise the results to all MOF materials.

\section{Conflicts of interest}

There are no conflicts to declare.

\section{Acknowledgements}

This project has received funding from the European Union's Horizon 2020 research and innovation programme under the 
Marie Sklodowska-Curie grant agreement No. 641887 (project acronym: DEFNET). Korean authors are grateful to the R\&D Convergence Program (CRC 14-1-KRICT) of National Research Council of Science \& Technology (NST) of the Republic of Korea. We would like to thank Prof. Sung June Cho (Chonnam National University) for his initial contribution to screening work on alumina binder in shaping of MOFs.

\section{Notes and references}

1 G. Maurin, C. Serre, A. Cooper and G. Férey, Chem. Soc. Rev., 2017, 46, 3104-3107.

2 F. Akhtar, L. Andersson, S. Ogunwumi, N. Hedin and L. Bergström, J. Eur. Ceram. Soc., 2014, 34, 1643-1666.

3 Advanced Processing and Manufacturing Technologies for Structural and Multifunctional Materials: A Collection of Papers presented at the 31st International Conference on Advanced Ceramics and Composites, January 21-26, 2007, Daytona Beach, Florida, ed. T. Ohji, M. Singh, J. A. Salem, D. Zhu and A. C. Society, Wiley-Interscience, Hoboken, N.J, 2008.

4 Activated Carbon Surfaces in Environmental Remediation, ed. T. J. Bandosz, Elsevier/AP, Amsterdam, 2006.

5 D. M. Ruthven, Principles of Adsorption and Adsorption Processes, Wiley, New York, 1984.

6 G. T. Whiting, A. D. Chowdhury, R. Oord, P. Paalanen and B. M. Weckhuysen, Faraday Discuss., 2016, 188, 369-386.

7 N.-L. Michels, S. Mitchell and J. Pérez-Ramírez, ACS Catal., 2014, 4, 2409-2417.

8 The Chemistry of Metal-Organic Frameworks: Synthesis, Characterization, and Applications, ed. S. Kaskel, Wiley-VCH Verlag GmbH \& Co. KGaA, Weinheim, 2016.

9 E. V. Ramos-Fernandez, M. Garcia-Domingos, J. JuanAlcañiz, J. Gascon and F. Kapteijn, Appl. Catal., A, 2011, 391, 261-267.

10 S. Aguado, J. Canivet and D. Farrusseng, Chem. Commun., 2010, 46, 7999.

11 D. Bazer-Bachi, L. Assié, V. Lecocq, B. Harbuzaru and V. Falk, Powder Technol., 2014, 255, 52-59.

12 T. Tian, J. Velazquez-Garcia, T. D. Bennett and D. FairenJimenez, J. Mater. Chem. A, 2015, 3, 2999-3005.

13 R. Semino, N. A. Ramsahye, A. Ghoufi and G. Maurin, ACS Appl. Mater. Interfaces, 2016, 8, 809-819.

14 T. Kitao, Y. Zhang, S. Kitagawa, B. Wang and T. Uemura, Chem. Soc. Rev., 2017, 46, 3108-3133.
15 N. Chanut, A. D. Wiersum, U.-H. Lee, Y. K. Hwang, F. Ragon, H. Chevreau, S. Bourrelly, B. Kuchta, J.-S. Chang, C. Serre and P. L. Llewellyn, Eur. J. Inorg. Chem., 2016, 4416-4423.

16 J. H. Cavka, S. Jakobsen, U. Olsbye, N. Guillou, C. Lamberti, S. Bordiga and K. P. Lillerud, J. Am. Chem. Soc., 2008, 130, 13850-13851.

17 A. D. Wiersum, E. Soubeyrand-Lenoir, Q. Yang, B. Moulin, V. Guillerm, M. B. Yahia, S. Bourrelly, A. Vimont, S. Miller, C. Vagner, M. Daturi, G. Clet, C. Serre, G. Maurin and P. L. Llewellyn, Chem.-Asian J., 2011, 6, 3270-3280.

18 P. Horcajada, S. Surblé, C. Serre, D.-Y. Hong, Y.-K. Seo, J.-S. Chang, J.-M. Grenèche, I. Margiolaki and G. Férey, Chem. Commun., 2007, 2820-2822.

19 J. W. Yoon, Y.-K. Seo, Y. K. Hwang, J.-S. Chang, H. Leclerc, S. Wuttke, P. Bazin, A. Vimont, M. Daturi, E. Bloch, P. L. Llewellyn, C. Serre, P. Horcajada, J.-M. Grenèche, A. E. Rodrigues and G. Férey, Angew. Chem., Int. Ed., 2010, 49, 5949-5952.

20 Y. Liu, J. F. Eubank, A. J. Cairns, J. Eckert, V. C. Kravtsov, R. Luebke and M. Eddaoudi, Angew. Chem., Int. Ed., 2007, 46, 3278-3283.

21 H. Chevreau, A. Permyakova, F. Nouar, P. Fabry, C. Livage, F. Ragon, A. Garcia-Marquez, T. Devic, N. Steunou, C. Serre and P. Horcajada, CrystEngComm, 2016, 18, 4094-4101.

22 N. Chanut, S. Bourrelly, B. Kuchta, C. Serre, J.-S. Chang, P. A. Wright and P. L. Llewellyn, ChemSusChem, 2017, 10, 1543-1553.

23 A. H. Valekar, K.-H. Cho, U.-H. Lee, J. S. Lee, J. W. Yoon, Y. K. Hwang, S. G. Lee, S. J. Cho and J.-S. Chang, RSC Adv., 2017, 7, 55767-55777.

24 J. Liu, J. Tian, P. K. Thallapally and B. P. McGrail, J. Phys. Chem. C, 2012, 116, 9575-9581.

25 J. Liu, Y. Wang, A. I. Benin, P. Jakubczak, R. R. Willis and M. D. LeVan, Langmuir, 2010, 26, 14301-14307.

26 A. C. Kizzie, A. G. Wong-Foy and A. J. Matzger, Langmuir, 2011, 27, 6368-6373.

27 H. Furukawa, F. Gándara, Y.-B. Zhang, J. Jiang, W. L. Queen, M. R. Hudson and O. M. Yaghi, J. Am. Chem. Soc., 2014, 136, 4369-4381.

28 J. Canivet, A. Fateeva, Y. Guo, B. Coasne and D. Farrusseng, Chem. Soc. Rev., 2014, 43, 5594-5617.

29 P. Ghosh, Y. J. Colón and R. Q. Snurr, Chem. Commun., 2014, 50, 11329-11331. 